\title{
Chloroquine in idiopathic pulmonary haemosiderosis
}

National Heart and Lung

Institute and Royal

Brompton National

Heart and Lung

Hospital, Sydney St,

London SW3 6NP,

Department of

Paediatrics

A Bush

J O Warner

Department of Lung

Pathology

M N Sheppard

Correspondence to:

Dr Bush.

Accepted 8 January 1992

Andrew Bush, Mary N Sheppard, J O Warner

\begin{abstract}
Two cases of idiopathic pulmonary haemosiderosis are reported in order to highlight the diagnostic problems, associated features, and the response to chloroquine, which is a new treatment for this life threatening condition.
\end{abstract}

Iron deficiency anaemia is common in childhood and usually dietary in origin. ${ }^{1}$ If a deficient intake is not the cause, diagnostic attention normally focuses on the gastrointestinal tract. Respiratory causes are rare and occult pulmonary haemorrhage is often not considered until other possibilities have been exhausted. ${ }^{2}$ We describe two children with primary pulmonary haemosiderosis in order to highlight some of the problems in making this diagnosis. We describe the response to chloroquine in order to draw attention to a novel treatment for this condition.

\section{Case reports}

CASE 1

When 4 years of age this previously well girl developed an erythema multiforme like rash which responded rapidly to oral steroids. Three months later the rash recurred, associated with cough, lethargy, and evidence of consolidation on the chest radiograph. The symptoms gradually remitted on steroids, but a high erythrocyte sedimentation rate (ESR) persisted. A year later she developed cough, weight loss, and dyspnoea. She was pale, a non-specific maculopapular rash was noted on the wrists and arms, and crackles were heard over both lung fields. Haemoglobin concentration was $62 \mathrm{~g} / \mathrm{l}$, with a hypochromic, microcytic picture, and the ESR was $82 \mathrm{~mm} /$ hour. The chest radiograph showed patchy shadowing in both lung fields. The serum iron concentration was $3.5 \mathrm{mmol} / \mathrm{l}$ (normal $11 \cdot 5-27 \cdot 0$ ) and total iron binding capacity $39 \mathrm{mmol} / \mathrm{l}$ (normal 36-77). A bone marrow biopsy specimen showed no stainable iron and no evidence of malignancy, and a jejunal biopsy was normal. The initial diagnosis of atypical pneumonia was changed to cryptogenic fibrosing alveolitis, and she was given oxygen, blood transfusion, and steroids. She made a good clinical response. She remained well until she developed localised scleroderma (morphoea) confirmed by biopsy at 7 years of age. She always complied poorly with treatment, and her steroids were finally stopped at age 11 years.

Three years later she developed a cough and wheeze. An initial diagnosis of asthma was made, but a flow volume loop was restrictive in type, and she was referred to the Royal Brompton Hospital. On admission, she was tachypnoeic, but there was no digital clubbing. There was widespread morphoea, sparing the face. She had fine end inspiratory basal crackles. The chest radiograph showed predominantly basal reticulonodular shadowing with prominent hilar lymphadenopathy (fig 1). Other investigations were haemoglobin concentration $96 \mathrm{~g} / \mathrm{l}$, with a hypochromic, microcytic picture; white cell count $12 \cdot 0 \times 10^{9} / 1$, platelets $581 \times 10^{9} / 1$, and ESR $126 \mathrm{~mm} /$ hour. Serum iron concentration was $7.5 \mathrm{mmol} / \mathrm{l}$, iron binding capacity 46 $\mathrm{mmol} / \mathrm{l}$, ferritin $202 \mu \mathrm{g} / \mathrm{l}$ (normal 15-300), and urea and electrolytes were normal. Lung function (percent predicted) results were: forced expiratory volume in one second $\left(\mathrm{FEV}_{1}\right) 31$, forced vital capacity (FVC) 29, total lung capacity (TLC) 66, residual volume 215 , and whole lung carbon monoxide transfer (TLCO) 27. At fibreoptic bronchoscopy the lower respiratory tract was macroscopically normal. The transbronchial biopsy specimen showed large numbers of haemosiderin laden macrophages within alveoli (fig 2). There was also haemosiderin impregnation of elastin in the alveolar wall and the walls of blood vessels. No other pathological features were seen in the specimen. These findings confirmed the clinical diagnosis of idiopathic pulmonary haemosiderosis. The bronchoalveolar lavage was technically unsatisfactory. She was started on chloroquine sulphate $200 \mathrm{mg}$ daily. Her ESR fell to $15 \mathrm{~mm} /$ hour, she required no further transfusions, her chest symptoms disappeared, and her chest radiograph cleared (fig 3). At age 16 she developed pyoderma gangrenosum.

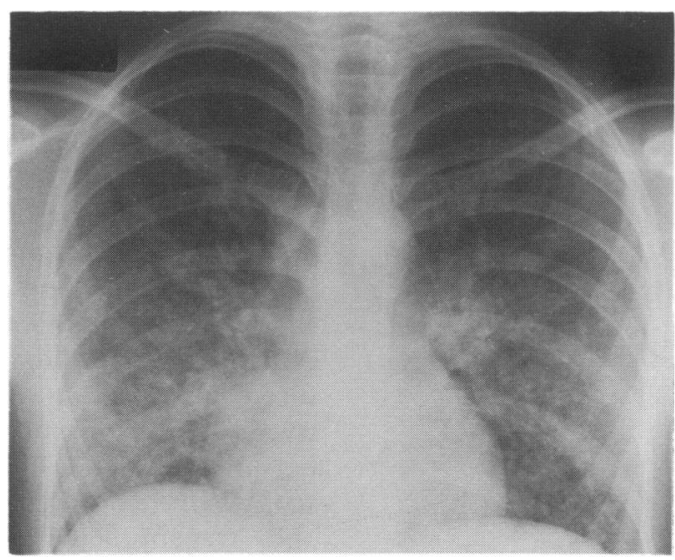

Figure 1 Chest radiograph of case 1 on admission to the Royal Brompton Hospital. There is prominent hilar lymphadenopathy with predominantly basal reticulonodular shadowing. 


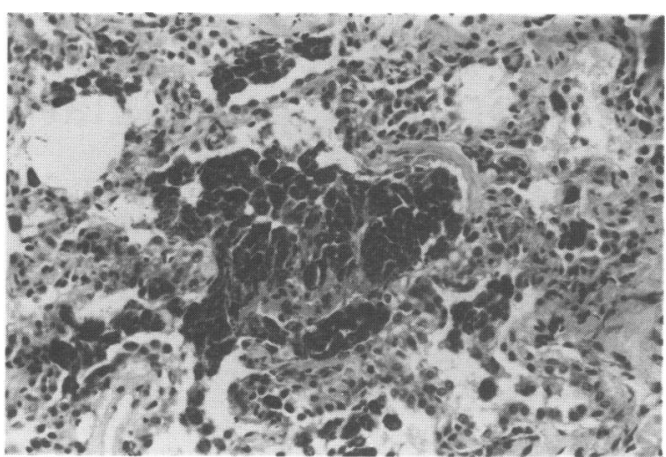

Figure 2 Transbronchial lung biopsy specimen of case 1 . Haematoxylin and eosin preparation showing dark haemosiderin within the cytoplasm of macrophages within alveoli (magnification $\times 400$ ).

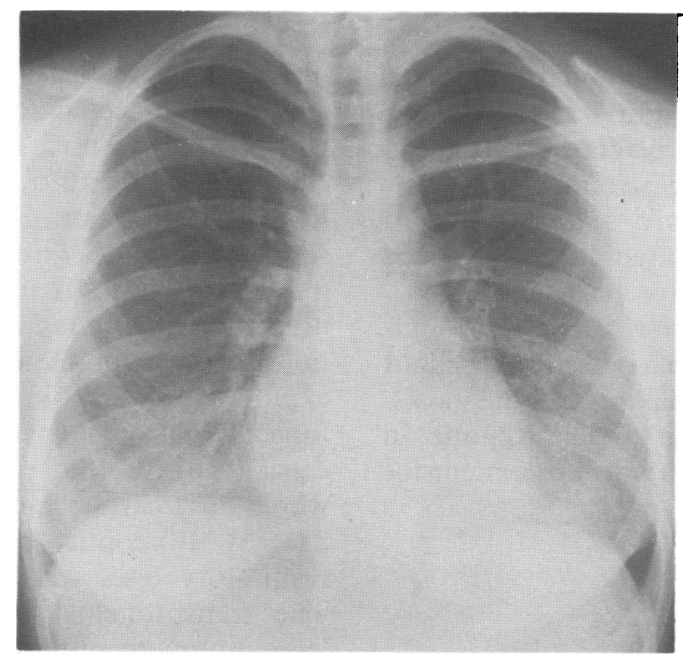

Figure 3 Chest radiograph of case 1 one year after starting chloroquine. There has been substantial clearing of the shadowing and regression of the lymphadenopathy.

Barium enema was normal. Now aged 17 years, she continues on chloroquine, and has had only mild relapses of pulmonary haemorrhage, which responded promptly to steroids. When last seen, her lung function had improved $\left(\mathrm{FEV}_{1} \quad 71 \%\right.$; FVC 86\%; TLC 92\%; TLCO $49 \%$ ) and her chest radiograph was nearly normal.

\section{CASE 2}

At 13 years of age this girl was found to have a profound iron deficient anaemia despite adequate intake and no evidence of excessive blood loss or malabsorption. Two years previously, at the time of an appendicectomy, her haemoglobin concentration had been normal. She was noted to have a cough and haemoptysis, with a chest radiograph reported as showing evidence of consolidation. Despite being transfused, her anaemia recurred, and further negative investigations included faecal occult bloods, barium meal and follow through, oesophagoscopy, bronchoscopy (bronchoalveolar lavage and transbronchial biopsy not performed), colonoscopy, jejunal biopsy, and rectal biopsy. Urinary haemosiderin and sediment and renal function were all normal. Ten months after her initial presentation the chest radiograph revealed

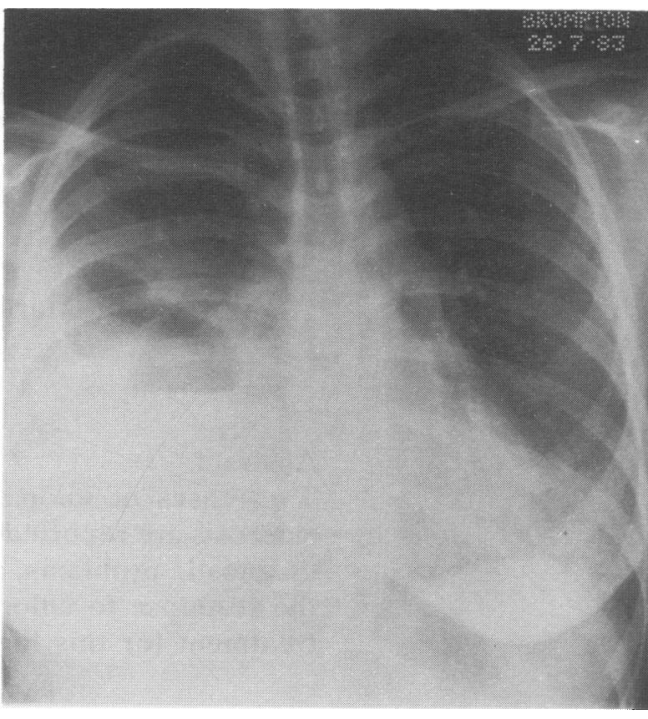

Figure 4 Chest radiograph of case 2 after her open lung biopsy. There are air-fluid levels in the right hemithorax completely obscuring the lung field. On the left there is basal reticulonodular shadowing. At right thoractomy a loculated pleural effusion was found.

granular shadowing, and the diagnosis of idiopathic pulmonary haemosiderosis was established by open lung biopsy. The procedure was complicated by a pneumothorax, and subsequently an encysted pleural effusion developed (fig 4). After decortication she was treated with prednisolone $60 \mathrm{mg}$ and azathioprine $100 \mathrm{mg}$ daily. She was also given high dose steroids by Nebuhaler, an empirical trial of a gluten free diet, and intensive plasmapheresis, but she continued to have haemoptyses and remained transfusion dependent (two units of packed cells every one to two months). Four years after her initial presentation she was started on chloroquine sulphate $200 \mathrm{mg}$ twice daily. Her other treatment was slowly withdrawn and she remained transfusion independent with only occasional very slight haemoptyses, one in association with influenza immunisation. Three years later chloroquine was discontinued, but within six months she had relapsed. Chloroquine $200 \mathrm{mg}$ daily was restarted and the disease remitted. However, retinal changes were detected during a routine ophthalmological check. These were reversed when chloroquine was discontinued. Unfortunately she has had further relapses and we were advised that she could not safely be treated with chloroquine again. Her renal function and antiglomerular basement membrane antibodies have remained normal. Currently she is receiving corticosteroids and cyclophosphamide to try to control her disease.

\section{Discussion}

These children are highly selected referrals to a tertiary centre and illustrate the difficulty of establishing the diagnosis of a rare cause of a common problem. Both children presented with iron deficiency anaemia, with only case 1 having a history of a previous respiratory illness, which in retrospect may have been due topulmonary haemosiderosis. The delay between first onset of symptoms and diagnosis was 10 
years in this child, and several months in the second case. During this time many unpleasant, invasive, and non-contributory investigations were performed, which might have been avoided if the diagnosis had been considered earlier.

Idiopathic pulmonary haemosiderosis can present at any age from the neonatal period to late adulthood. ${ }^{3-5}$ The usual presentation is with iron deficiency anaemia, which may initially respond to oral iron. ${ }^{3}$ The classical triad of unexplained anaemia, haemoptysis, and pulmonary infiltrates is rare in childhood, only partly because bloodstained sputum is usually swallowed. ${ }^{2}$ Half have no pulmonary symptoms. ${ }^{5}$ Swallowed blood may lead to positive faecal occult bloods, mimicking gastrointestinal bleeding. ${ }^{2}$ Rarer presentations are the febrile child with a bronchopneumonia like illness ${ }^{5}$ and acute fulminating pulmonary haemorrhage. ${ }^{24}$ The physical signs may include digital clubbing and hepatosplenomegaly, ${ }^{2}{ }^{3}$ with usually a clear chest to auscultation. ${ }^{6}$ However, other than pallor, the child may appear completely normal on examination. Investigations may show eosinophilia, ${ }^{3}$ and the chest radiograph may be normal, or show non-specific infiltrates, miliary shadowing, or confluent shadows. ${ }^{3}$ Hilar and mediastinal lymphadenopathy may be present. ${ }^{7}$ There is no correlation between pulmonary symptoms and radiographic abnormalities. ${ }^{7}$ Lung function tests show restrictive physiology and reduced carbon monoxide transfer at rest and on exercise in the chronic phase. ${ }^{3}$ In the acute phase carbon monoxide transfer rises by more than $30 \%$ above baseline and is associated with a fall in haemoglobin by $20-40 \mathrm{~g} / \mathrm{l}$ and deteriorating radiographic changes over a 24 to 48 hour period. ${ }^{8}$ Although the diagnosis can be made by finding haemosiderin laden macrophages in sputum or gastric washings, ${ }^{5}$ a more direct approach is fibreoptic bronchoscopy with bronchoalveolar lavage and transbronchial biopsy. ${ }^{9}$ Diagnosis can be established by open lung biopsy, but transbronchial biopsies should be interpreted cautiously, as haemosiderin laden macrophages within alveoli can be seen in other conditions including heart failure, pneumonia, primary pulmonary hypertension, and pulmonary veno-occlusive disease. ${ }^{10}$ The clinical history is thus of vital importance to the pathologist. Unusual features in our cases include the association with erythema multiforme, morphoea, and pyoderma gangrenosum (case 1) and relapse in association with immunisation (case 2), neither of which has to our knowledge been described before.

The variable course and rarity of the disease makes assessment of treatment difficult; for example, a third child referred to us in the same period went into spontaneous remission (details not given). We elected to use chloroquine because of its lack of toxicity compared with immunosuppressive regimens that have been used $^{11}$ and because of isolated reports of a dramatic response in childhood cryptogenic fibrosing alveolitis. ${ }^{12}$ Unfortunately, as in case 2 , the use of chloroquine may be limited by retinal toxicity and regular ophthalmic checks are essential.

In a condition that shows spontaneous fluctuations in severity, it is difficult to be certain of the therapeutic benefit of any intervention. In case 2, a prompt decrease in the severity of her illness on starting chloroquine, together with a history of relapse on each of two occasions when chloroquine was stopped, strongly supports a real beneficial effect. Case 1 is more difficult to assess, in part because of poor compliance with medication, but it would seem probable that her improvement was related to chloroquine treatment.

In summary, we describe two cases of childhood idiopathic pulmonary haemosiderosis, one of whom also had several unusual skin rashes. We highlight the diagnostic difficulties and delays and describe our experience with chloroquine as a treatment for this disease. We suggest that a therapeutic trial of chloroquine should be considered in children with idiopathic pulmonary haemosiderosis.

1 Illingworth RS. Common symptoms of disease in childhood. 9th Ed. Oxford: Blackwell Scientific Publications, 1988.

2 Matsaniotis N, Karpouzas J, Apostolopoulou E, Messaritakis J. Idiopathic pulmonary haemosiderosis in children. Arch Dis Child 1968;43:307-9.

3 Repetto G, Lisbon C, Emparanza E, et al. Idiopathic pulmonary hemosiderosis: clinical, radiological, and respiratory function studies. $\mathcal{F}$ Pediatr 1967;40:24-32

4 Nickerson $\mathrm{HJ}$. Idiopathic pulmonary hemosiderosis in a fivemonth-old infant. Clin Pediatr (Phila) 1968;7:416-8.

5 Kjellman B, Elinder G, Garwic\% S, Swan H. Idiopathic pulmonary haemosiderosis in Swedish children. Acto Paediatr Scand 1984;73:584-8.

6 Seeler RA. Pediatric perplexities: idiopathic pulmonary hemosiderosis anemia and 'pneumonia'. Illinois Medical fournal 1973; 144:421-4.

7 Snider GL, Mark EJ. Case records of the Massachussetts General Hospital No 30-1988. N Engl F Med 1988;319: General

8 Ewan PW, Jones HA, Rhodes CG, Hughes JMB. Detection of intrapulmonary hemorrhage with carbon monoxide uptake. $N$ Engl I Med 1976;295:1391-6.

9 Drew WL, Finley TN, Golde DW. Diagnostic lavage and occult pulmonary hemorrhage in thrombocytopenic immunocompromised patients. Am Rev Respir Dis 1977 116:215-21.

10 Travis WD, Colby TV, Lombard C, Carpenter H. A clinicopathologic study of 34 cases of diffuse pulmonary hemorrhage with lung biopsy confirmation. Am $\mathcal{f}$ Surg Pathol 1990;14:1112-25.

11 Pozo-Rodriguez F, Freire-Campo JM, Gutierre\%-Millet V, Barbosa-Ayucar C, Diaz. De Atauti J, Martin-Escribano P. Idiopathic pulmonary haemosiderosis treated by plasmapheresis. Thorax 1980;35:399-400.

12 Leahy F, Pasterkamp H, Tal A. Desquamative interstitial pneumonia responsive to chloroquine. Clin Pediatr (Phila) 1985;24:230-2. 